\title{
The thermospheric auroral red line polarization: confirmation of detection and first quantitative analysis
}

\author{
Jean Lilensten ${ }^{1, *}$, Mathieu Barthélémy ${ }^{1}$, Pierre-Olivier Amblard ${ }^{2}$, Hervé Lamy ${ }^{3}$, Cyril Simon Wedlund ${ }^{3}$, \\ Véronique Bommier ${ }^{4}$, Joran Moen ${ }^{5}$, Hanna Rothkaehl ${ }^{6}$, Julien Eymard ${ }^{1}$, and Jocelyn Ribot ${ }^{1}$ \\ 1 UJF-Grenoble 1/CNRS-INSU, Institut de Planétologie et d'Astrophysique de Grenoble (IPAG), UMR 5274, \\ 38041 Grenoble, France \\ *corresponding author: e-mail: jean.lilensten@obs.ujf-grenoble.fr \\ 2 Gipsa-Lab, 38041 Saint-Martin d'Hères Cedex 9, France \\ 3 Belgian Institute for Space Aeronomy, Ringlaan-3-Avenue Circulaire, B-1180 Brussels, Belgium \\ 4 LESIA, CNRS, Observatoire de Paris, 92190 Meudon, France \\ 5 Department of Physics, University of Oslo, P.O. Box 1048, N-0316 Blindern, Oslo, Norway \\ 6 Space Research Centre Polish Academy of Sciences, ul.Bartycka 18a, 00-716 Warsaw, Poland \\ Received 18 February 2012 / Accepted 21 December 2012
}

\begin{abstract}
The thermospheric atomic oxygen red line is among the brightest in the auroral spectrum. Previous observations in Longyearbyen, Svalbard, indicated that it may be intrinsically polarized, but a possible contamination by light pollution could not be ruled out. During the winter 2010/2011, the polarization of the red line was measured for the first time at the Polish Hornsund polar base without contamination. Two methods of data analysis are presented to compute the degree of linear polarization (DoLP) and angle of linear polarization (AoLP): one is based on averaging and the other one on filtering. Results are compared and are in qualitative agreement. For solar zenith angles (SZA) larger than $108^{\circ}$ (with no contribution from Rayleigh scattering), the DoLP ranges between 2 and 7\%. The AoLP is more or less aligned with the direction of the magnetic field line, in agreement with the theoretical predictions of Bommier et al. (2010). However, the AoLP values range between $\pm 20^{\circ}$ around this direction, depending on the auroral conditions. Correlations between the polarization parameters and the red line intensity $I$ were considered. The DoLP decreases when $I$ increases, confirming a trend observed during the observations in Longyearbyen. However, for small values of $I$, DoLP varies within a large range of values, while for large values of $I$, DoLP is always small. The AoLP also varies with the red line intensity, slightly rotating around the magnetic field line.
\end{abstract}

Key words. thermosphere - auroral emissions - polarization

\section{Introduction}

In the nightside ionospheric $F$ region, typically from 150 to $400 \mathrm{~km}$, the main source of ionization and excitation is the particle precipitations of solar/magnetospheric origin. This region is collisional, with elastic as well as inelastic collisions. Chemical reactions including both neutral and ionized species also take place at these altitudes. The thermospheric emissions come from the deactivation of excited atoms, molecules, or ions. The atomic oxygen is one of the main contributors to the glow in the visible light. Its main emissions correspond to transitions from state ${ }^{1} \mathrm{~S}$ to ${ }^{1} \mathrm{D}$, and ${ }^{1} \mathrm{D}$ to ${ }^{3} \mathrm{P}$. The first transition gives rise to the green line at $557.7 \mathrm{~nm}$, and the second one forms the triplet at $630.0,636.4$, and $639.2 \mathrm{~nm}$, which constitutes the red line emission. The production of the excited states $\mathrm{D}$ is due to different phenomena (Witasse et al. 1998 and references therein): energetic electron impact, photodissociation, chemical reaction with $\mathrm{N}\left({ }^{2} \mathrm{D}\right)$ or $\mathrm{N}^{+}$, cascade from an upper level, and dissociative recombination with $\mathrm{O}_{2}^{+}$. The photodissociation, the chemical reactions, or the cascade from upper energy levels is isotropic. Isotropy also happens with the dissociative recombination when thermal electrons are involved, because the thermal distribution is spatially isotropic (maxwellian). Therefore, in the diurnal case when the source is the electromagnetic Extreme Ultraviolet
(EUV) radiation, no polarization of the oxygen emission is expected. At night, when the main source is energetic particle impact, the presence of a magnetic field imposes a collimated pitch angle. In this case, emission could be polarized because the excitation source is anisotropic. This happens during nighttime with a larger probability when the energies of the incoming electrons are relatively homogeneous. The polarization of the thermospheric atomic oxygen red line at $630 \mathrm{~nm}$ has been discovered recently (Lilensten et al. 2008). During this campaign, the emission was observed with a dedicated spectro-photo-polarimeter (SPP) placed in the Svalbard Archipelago $\left(78^{\circ} \mathrm{N}\right.$ latitude). Observations were made only in one direction, namely to the North, such that the line of sight was approximately perpendicular to the magnetic field line at an altitude of $210 \mathrm{~km}$. In a previous paper (Lilensten et al. 2006), prevision, it was foreseen that the angle of polarization should be perpendicular to the line of sight when looking perpendicular to the magnetic field. Morever, $210 \mathrm{~km}$ corresponds to the altitude where the red line is expected to be the most intense (Johnsen et al. (2012) and reference therein). During the following campaigns, we were able to demonstrate that a southward or a parallel to the magnetic field (B) pointing reduces drastically the degree of linear polarization (Barthélemy et al. 2011). 
All these observations were carried out at the Kjell Henriksen Observatory (KHO) optical facility. Unfortunately, when pointing north, the line of sight was very close to the city of Longyearbyen. As a consequence, fully reliable values of the polarization parameters could not be derived because of a possible contamination by light pollution. Therefore, the instrument was moved to the Polish Hornsund polar base in summer 2010 and observations were carried out during winter 2010/2011. The goals of this campaign were:

- To definitely rule out light pollution as partly responsible for the polarization observed during the first measurements.

- To provide reliable polarization parameters: degree (DoLP) and angle of linear polarization (AoLP).

- To start examining whether the DoLP and/or AoLP variations are related to the geomagnetic activity in order to use them as proxies in space weather applications.

The first goal has been reached and constitutes the core of this article. With the use of uncontaminated datasets and a more accurate data processing, the polarization parameters have been better estimated (second goal). Unfortunately the third goal could not be fulfilled. Indeed, due to harsh polar conditions, a major breakdown of the SPP occurred after a few weeks of measurements. During these weeks solar and geomagnetic activity were very low and constant, preventing us from seeing how the polarization parameters vary.

In this paper, the AoLP is measured in a reference frame such that $0^{\circ}$ corresponds to the vertical, and $90^{\circ}$ to the horizontal. Angles are counted positively clockwise.

\section{Presentation of the Hornsund campaign}

\subsection{The spectro-photo-polarimeter (SPP)}

The SPP includes two detection channels with identical photomultipliers, front lenses, and $1 \mathrm{~nm}$ width interference filters centered at $630.0 \mathrm{~nm}$. The field of view is $2^{\circ}$. A linear polarizing filter rotates in front of the first channel (referred to as the polarized channel) with a $4.02 \mathrm{~s}$ period and with a sampling rate of 20 points per second. This rotation period was chosen after considering the natural life time of the $\mathrm{O}\left({ }^{1} \mathrm{D}\right)$ state (about $110 \mathrm{~s}$ ) because the modulation had to be small with respect to this time. Moreover, the design of a device with front optics rotating in $4 \mathrm{~s}$ was technically feasible for an instrument operating under harsh conditions. The other channel, which has no polarizing filter, is referred to as the "reference channel". The photomultiplier is cooled with a regulated Peltier device, which ensured low values of the dark current during the whole campaign. Dark current was regularly measured and despite the cold Hornsund conditions, it was stable with an average of 4 counts per angle position of the polarizer in the polarized channel, and 2 in the reference channel.

The photomultiplier was used in photon counting mode, i.e. the device was only counting pulses above some discrimination level without measuring the height of these pulses (Hamamatsu Technical Information 1998). In this mode, the number of signal pulses $N$ counted for a certain period of time exhibits a temporal fluctuation that can be approximated by a Poisson distribution. The amplifier noise can be ignored by using a sufficiently high value of the discrimination level. Therefore in the following, intensities will be given in the number of counts.

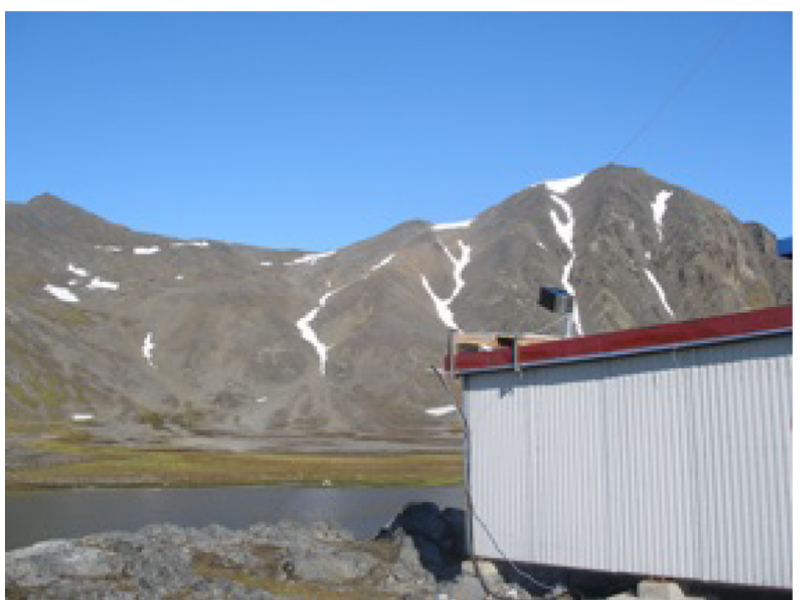

Fig. 1. The position of the instrument on the roof of a cabin for the winter campaign.

\subsection{The Hornsund polar base}

The SPP was installed at the Hornsund Polish base, located at $77.0^{\circ} \mathrm{N}$ and $15.33^{\circ} \mathrm{E}$. This polar station of the Institute of Geophysics, Polish Academy of Sciences, is situated on south Spitsbergen, near the entrance to Hornsund fjord, on its northern shore, at Isbjrnhamna (Polar Bear Bay), on a flat marine terrace, $10 \mathrm{~m}$ above sea level. Poland carries out research on Svalbard as one of the countries that signed the Spitsbergen Treaty - the international agreement setting the status of Spitsbergen archipelago in 1921.

In order to avoid any polarization introduced by an astronomical transparent dome, the instrument was installed on the roof of a scientific cabin located $500 \mathrm{~m}$ away from the main station, pointing toward the magnetic north with an elevation of $23.0^{\circ}$. This ensures both to point above the northward mountain and to be approximately perpendicular to the magnetic field line at about $210 \mathrm{~km}$ height. Figure 1 shows the location of the SPP: there is no parasitic light in the pointing direction and the main station is too far away to the south to create any contamination. Finally, the experiment was run during moonless nights to avoid polarization induced by the moon light diffusion, in particular on the snow covering the landscape.

The advantage of observing at a high latitude is the location in the polar cap, where electrons with a mean energy of several hundreds of electronvolts constantly precipitate in the upper atmosphere, called polar rain (Fairfield \& Scudder 1985). In some cases, this energy reaches a few keV (Newell \& Meng 1990). The red line is therefore permanently emitted in the upper atmosphere. At such high latitude, another source for the red line emission exists: the recombination of transpolar cap patches that gives rise to optical emissions in the order of hundreds of Rayleigh (depending on the solar activity; Lorentzen et al. 2004; Moen et al. 2007; Moen et al. 2008).

\subsection{Calibration of the SPP}

Photomultipliers may be anisotropic devices, detecting more intensity in one linear polarization direction than the other, hence introducing instrumental polarization. The instrumental polarization has been measured in laboratory using a calibration lamp and a reflection on a diffuse lambertian white screen. DoLP was $0.9 \%$ with an AoLP of $16.9^{\circ}$. This calibration has 


\section{J. Lilensten et al.: Polarization of the auroral red line}

been performed in laboratory conditions at room temperature, which is very different from the outside temperature conditions during the measurements. In the following, it is assumed that the large differences in temperature do not modify significantly the instrumental polarization. In the future, the variation of the instrumental polarization with temperature will be calibrated in climatic chambers.

To be sure that data from the SPP were not contaminated by any light pollution, the following test was performed. On December 7 at 17:28 LT, all the lamps of the polar station were turned off during $8 \mathrm{~min}$. The next day, all the lamps were turned on from 7:40 to 8:20 LT. During these periods, no particular variation could be noticed in the polarimetric data.

\subsection{Environmental conditions}

A detailed report on the environmental conditions is given in Appendix A. We selected data when the sky was perfectly clear both in Hornsund and in Longyearbyen, $150 \mathrm{~km}$ North. The solar activity was mainly at very low levels and the geomagnetic activity was predominantly quiet for the entire period.

\section{Methods of analysis}

During the campaign, due to the harsh polar conditions (temperature, wind), the quality of the data obtained with the SPP progressively deteriorated. A number of spurious spikes appeared in the data. Before processing the data, the first step consisted in removing these spikes. The method is described in Appendix B.

With a rotating polarizing filter, the variation of the measured intensities with the position angle of the filter should follow the Malus law:

$$
f(\alpha)=A \cos ^{2}(\alpha-\theta)+B
$$

where $\alpha$ is the angle of the polarizer, $A$ is the amplitude of the variation, $B$ is the offset, and $\theta$ is the angle corresponding to the maximum of the variation (see Fig. 2). Due to statistical errors, a non-linear fit must then be applied to the data to find the parameters $A, B$, and $\theta$ that minimize a sum of the squared errors (Lagarias et al. 1998). The DoLP and AoLP are related to the fitted parameters via:

$$
\operatorname{DoLP}=\frac{I_{\max }-I_{\min }}{I_{\max }+I_{\min }}=\frac{A}{2 B+A}
$$

$$
\mathrm{AoLP}=\theta
$$

During a full rotation of the filter (4.02 s), we assume that the polarization of the red line is constant. Measuring polarization with a better time resolution is out of reach with the SPP. But during the $4.02 \mathrm{~s}$ time interval, intensities of the red line emission can also vary, affecting the measurement of the polarization. We call $\bar{I}_{\text {pol }}$ the average intensity measured over the $4.02 \mathrm{~s}$ in the polarized channel and $\bar{I}_{\text {ref }}$ the one in the reference channel. At any time $t$ during the considered $4.02 \mathrm{~s}$, the intensity in the polarized channel is scaled after:

$$
I_{\mathrm{pol}}^{\mathrm{norm}}(t)=I_{\mathrm{pol}}(t) \times \frac{\bar{I}_{\mathrm{ref}}}{\bar{I}_{\mathrm{pol}}},
$$

$I_{\mathrm{pol}}^{\text {norm }}$ represents the normalized intensity used in the following data processing.

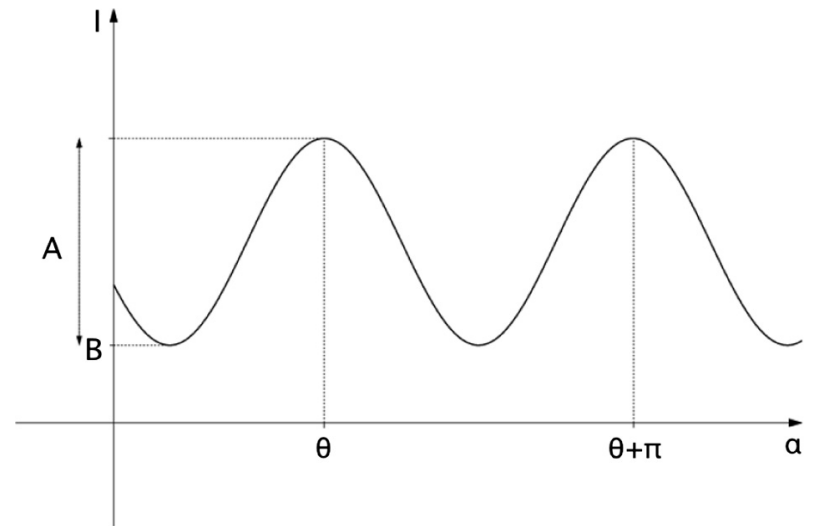

Fig. 2. Typical variation of the intensity versus the angle of the polarizer, expected from the Malus law.

According to Foord et al. (1969) and to the Hamamatsu technical note, the signal-to-noise ratio (SNR) was estimated during the whole process by:

$$
\mathrm{SNR}=\frac{N_{\mathrm{S}}}{\sqrt{N_{\mathrm{S}}+2 N_{\mathrm{DC}}}} \sqrt{t}
$$

where $N_{\mathrm{S}}$ represents the number of counts per second, $N_{\mathrm{DC}}$ is the number of counts in dark current conditions, and $t$ is the integration time.

Two methods of analysis were used to process the data, one using averaging techniques, the other using filtering techniques.

\subsection{Data processing using averaging techniques}

This processing method consists first in adding data from $N$ consecutive polarizing filter rotations and in averaging data for each position angle of the polarizer to obtain a good SNR. In this work, a value of $N$ equal to 8 was used as a compromise between good SNR and a reasonable time resolution (about $32 \mathrm{~s}$ ). Then a moving average on 5 points was applied to smooth out rapid fluctuations and highlight the cosine squared variation expected from the Malus law.

For the non-linear fit, several starting points in the parameter space are tested to avoid finding local minima of the sum of the squared error. The final parameters are the values of $A, B$, and $\theta$ that lead to the absolute minimum of the sum of the squared error. An estimation of the uncertainties on these parameters is obtained assuming that the parameters are distributed normally and are independent. Then these errors are propagated in Eq. (2) to obtain $\sigma_{\text {DoLP }}$, the uncertainty on DoLP:

$$
\sigma_{\mathrm{DoLP}}^{2}=\sigma_{A}^{2} \frac{4 B^{2}}{(2 B+A)^{4}}+\sigma_{B}^{2} \frac{4 A^{2}}{(2 B+A)^{4}} \text {. }
$$

Figure 3 gives an example of the fitting with the averaging method.

The influence of the two averaging processes on the determination of the polarization parameters was checked. The AoLP is not too affected but the DoLP is slightly underestimated. For example, during the measurement of the instrumental polarization with the unpolarized calibration lamp, the DoLP measured when averaging was $0.03 \%$ lower than without averaging.

The last step is to correct for the instrumental polarization. For that, the SPP (photo-multiplier + polarizing filter) is 


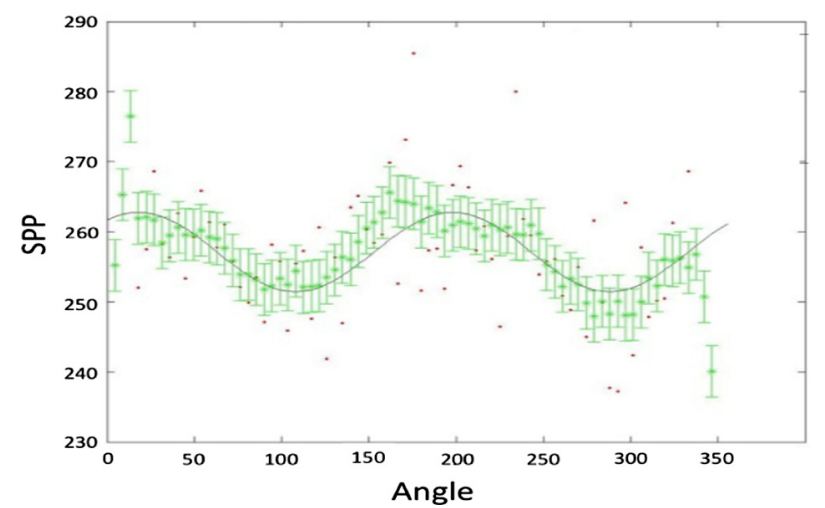

Fig. 3. Example of the fitting with the averaging method (here on November 8,2010 , at 1:13:56). The horizontal axis is $\alpha$, the angle (in degrees) of the polarizing filter, and the vertical axis is the intensity of the red line in number of counts $N_{\mathrm{S}}$ per second. The red dots represent the raw data. The green stars are the averaged data on eight consecutive turns of the polarizing filter. The error bars are calculated as $\sqrt{t\left(N_{\mathrm{S}}+2 N_{\mathrm{DC}}\right)}$. The continuous line is the best fit, providing a DoLP equal to $2.2 \pm 0.2$, and an AoLP equal to $18^{\circ}$.

considered as a partial polarizer and the Mueller formalism is used to compute the true (or real) DoLP and AoLP (see Appendix C). For the instrumental polarization, we used $0.9 \%$ for the DoLP, and $16.9^{\circ}$ for the AoLP.

\subsection{Data processing using filtering techniques}

The data processing has been explained by Lilensten et al. (2008) and further developed by Barthélemy et al. (2011). However, it is constantly improving, so that it is explained below. First, the dark current is corrected. Then two different filters are applied.

Next, the data are filtered with a low-pass filter at $1.5 \mathrm{~Hz}$. The choice of the cut-off frequency is based on the fact that the polarization filter rotates at $0.25 \mathrm{~Hz}$. If a polarized signal is present, intensity must exhibit two minima/maxima, hence a $0.5 \mathrm{~Hz}$ signal. In Figures 4 and 5, examples of spectrograms for the polarized channel and the reference channel are shown. A signal at approximately $0.5 \mathrm{~Hz}$ is clearly visible in the polarized channel when the intensity of the aurora strongly increases. At the same time no similar signal can be seen in the reference channel. The signal at $0.5 \mathrm{~Hz}$ is definitely present at all times but cannot be seen in the figure because of the color scale. The same features are observed in all data obtained during this campaign.

To detect polarization in the spectrograms, the following method is used: only data with a maximum in the power spectral density (PSD) between 0.4 and $0.6 \mathrm{~Hz}$ and a probability density larger than $20 \%$ are kept. Indeed, if the signal is pure noise, the maximum in the PSD can occur anywhere in the spectrogram between 0 and $1.5 \mathrm{~Hz}$. Hence the probability that this maximum is located between 0.4 and $0.6 \mathrm{~Hz}$ is $\sim 13.3 \%$ $\left(=\frac{0.6-0.4}{1.5}\right)$. Any maximum detected with a probability density close to this value was then considered as due to noise. Alternatively, a maximum detected in this frequency range with a probability density larger than or equal to $20 \%$ was considered as a polarized signal. Since a $0.5 \mathrm{~Hz}$ polarized signal should not appear in the PSD of the reference channel, this channel was used to check that no spurious signal was observed.

After this first test ensuring that the polarization detected is not due to noise, the raw data are filtered with a bandpass filter

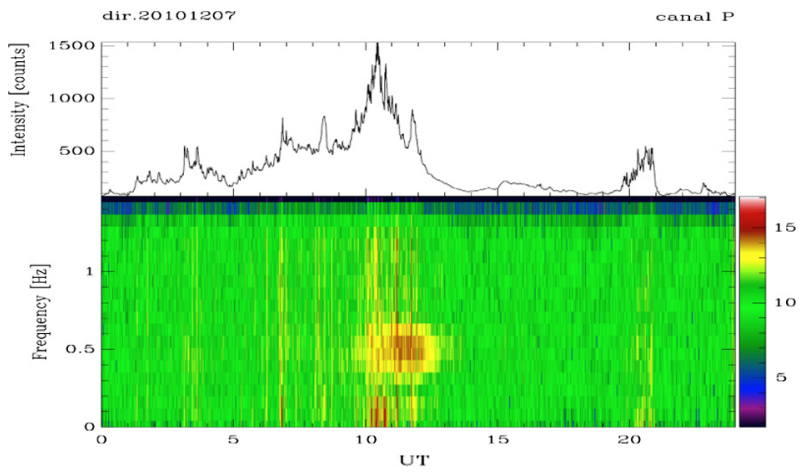

Fig. 4. Upper panel: intensity of the oxygen red line measured in the polarized channel and normalized to the one measured in the Reference channel in arbitrary units (photon counts). Lower panel: spectrogram of the signal.

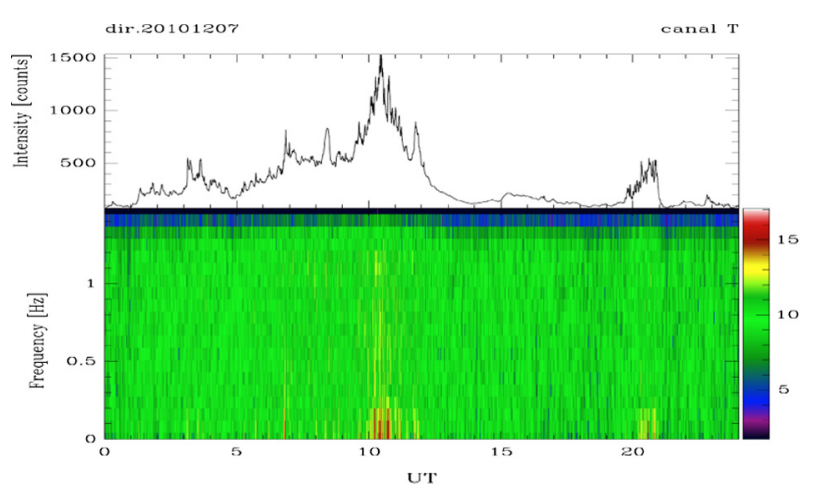

Fig. 5. Same as in Figure 4 for the reference channel.

centered at $0.5 \mathrm{~Hz}$ and with a bandwidth of $0.5 \mathrm{~Hz}$. An inverse Fourier transform is applied and each cycle measurement is fitted with the cosine squared function (Eq. 1). A LevenbergMarquardt method was used (Press et al. 1992). An example of the fitting procedure is given in Figure 6. The error bars are not provided because they have no meaning once the data are filtered. Indeed, the noise on the raw data is scattered over the whole frequency spectrum. The filtering results in its elimination at all the frequencies, but $0.5 \mathrm{~Hz}$. In order to study the propagation of the noise on the raw data on the polarization parameters, a random noise generator should be used. This is out of scope of this paper mainly because of the cpu time it would necessitate. Theoretically, there should be $30 \times 80=2400$ runs for each polarizer cycle since the noise is independent from a point to the other. However, it should not introduce any significant difference in the results, considering the discussion above.

The influence of the gain of the filter on the values of the DoLP was checked. When using the unpolarized calibration lamp, the values of the DoLP found with this method are in excellent agreement with those obtained with the method based on averaging, giving confidence in the method. The instrumental polarization was corrected using again the Mueller formalism. The main advantage of the method using filtering is that it provides a time resolution equal to that of the instrument (4 s).

With this series of steps in the signal processing, we believe that the results are as close as they can be to calibrated polarization values with this instrument. 


\section{J. Lilensten et al.: Polarization of the auroral red line}

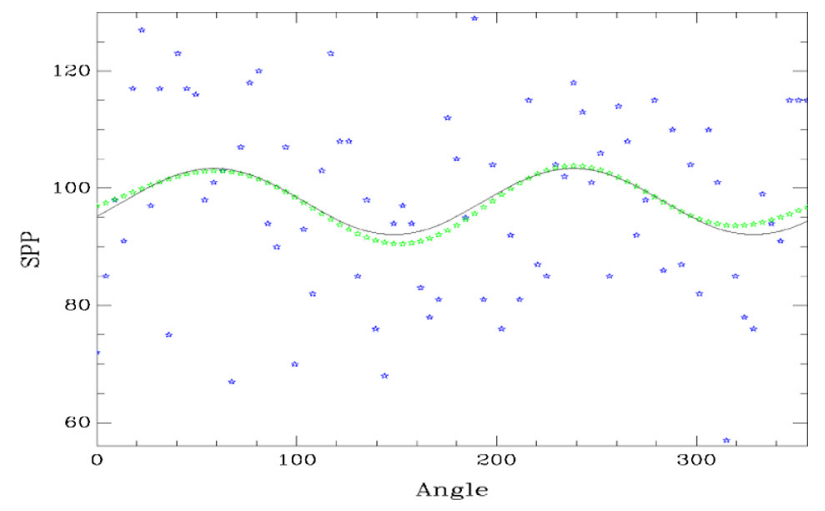

Fig. 6. Example of the fitting with the filtering procedure. The absissa represents the angles and the vertical axis is the number of counts. The blue stars represent the raw data. The green stars are the result of the filtering around $0.5 \mathrm{~Hz}$. The continuous line is the result of the fitting procedure.

\section{Results}

During this campaign, only observations with the SPP pointing northward (perpendicular to the magnetic field line at $210 \mathrm{~km}$ ) are available. Indeed, observations with the SPP pointing southward (parallel to the magnetic field line) were planned in the schedule after January. Unfortunately, they could not be carried out since meanwhile the instrument broke down.

From the whole set of data, six periods were identified with continuous data. The lack of data during other periods was due to either the presence of clouds or because of power failures. The periods with available data are summarized in Table 1.

The values of the probability densities contained in the 0.4 $0.6 \mathrm{~Hz}$ range are given in Table 2 for both channels and for each day of observation. They are remarkably stable in the reference channel. In the polarized channel, on November 9 and December 9, the values of the PSD in the polarized channel are close to the one expected from noise. Therefore, these data must be considered with an extreme care. For comparison, during the observations in Longyearbyen (Barthélemy et al. 2011), the values were smaller in the polarized channel (around 20\%) and varied around $13 \%$ in the reference channel. The measurements in the polarized channel could indicate an effect of the light pollution of the nearby city.

The results of the six periods are shown in Figures 7 to 12, where the abscissa is given in local time $(=\mathrm{UT}+1.0553)$ and a vertical line is plotted at midnight. The quantities plotted are averaged over $3 \mathrm{~min}$. In these figures, the error bars on the intensities are plotted according to Eq. (5). Sunlight scattering from the upper atmosphere (Ugolnikova et al. 2004; Cronin et al. 2006) occurs for solar zenith angles smaller than $108^{\circ}$ and creates a large polarization by Rayleigh scattering. To visualize the periods with and without sunlight scattering, the solar zenith angle is plotted in the upper panel of each figure and a horizontal dashed line shows the $108^{\circ}$ limit.

\subsection{Degree of linear polarization (DoLP)}

As explained in the previous section, two different methods of analysis were applied on the data, one relying on averaging, the other on filtering. A comparison of the DoLP measurements obtained with the unpolarized calibration lamp in laboratory ensures that the two methods give similar values of the DoLP.
Table 1. The six periods extracted from the dataset. Times are in UT and in decimal hours.

\begin{tabular}{lcccr}
\hline \hline Period & Start day & Start UT & End day & End UT \\
\hline 1 & Nov. 7 & 16.48 & Nov. 8 & 3.00 \\
2 & Nov. 8 & 14.30 & Nov. 8 & 24.00 \\
3 & Nov. 9 & 16.30 & Nov. 10 & 7.44 \\
4 & Dec. 2 & 18.89 & Dec. 3 & 6.83 \\
5 & Dec. 5 & 10.92 & Dec. 9 & 3.00 \\
6 & Dec. 10 & 0.00 & Dec. 11 & 7.99 \\
\hline
\end{tabular}

Table 2. Percentage of power spectral density data contained in the interval $0.4-0.6 \mathrm{~Hz}$ over the full night.

\begin{tabular}{ccc}
\hline \hline Date & Polarized channel & Reference channel \\
\hline Nov. 7 & 20.9 & 11.9 \\
Nov. 8 & 27.9 & 12.7 \\
Nov. 9 & 14.3 & 12.3 \\
Nov. 10 & 32.1 & 11.2 \\
Dec. 2 & 18.0 & 12.5 \\
Dec. 3 & 20.9 & 11.7 \\
Dec. 5 & 30.5 & 11.9 \\
Dec. 6 & 27.3 & 11.4 \\
Dec. 7 & 32.1 & 11.0 \\
Dec. 8 & 30.7 & 10.3 \\
Dec. 9 & 15.4 & 11.5 \\
Dec. 10 & 28.8 & 11.4 \\
Dec. 11 & 20.6 & 10.0 \\
\hline
\end{tabular}

The averaging method underestimates a bit the DoLP for signals with large SNR. The filtering method provides a DoLP with a much better time resolution.

An example of the comparison between the two methods is shown in Figure 9. The values of the DoLP have a similar behavior. During the day, when the solar zenith angle (SZA) is smaller than $108^{\circ}$, the polarization is large and due to Rayleigh scattering of the sunlight by the upper atmosphere. The signal is stationary with regard to the 4 s rotation of the polarizer. The values of the DoLP obtained by the two methods are nearly identical. On the other hand, at night (when the SZA is larger than $108^{\circ}$ ), those obtained with the averaging method are systematically smaller than those obtained with the filtering method. This is due to a bias introduced by averaging a non-stationary signal. For the other periods, only the values provided by the filtering method are shown in the plots for clarity.

During daytime, very large values of the DoLP (up to 8$10 \%$ in some cases) are observed due to Rayleigh scattering. This can be seen at the start of period 2, at the end of period 3 , and during periods 5 and 6 . In period 5 (covering four consecutive days), it is obvious that the sunlight polarization is different from one day to the other. These differences are probably due for a large part to the aerosols. This component is difficult to evaluate because scattering from aerosols is not Rayleigh scattering nor Mie scattering as the aerosols are generally not spherical. There may also be a component related to the geomagnetic activity. To retrieve such a component, a modeling of the Rayleigh scattering by the aerosols would be needed and this is out of scope of this paper. At night, especially around midnight, there is a clear anti-correlation between the DoLP and the intensity of the red line. This can be clearly seen during period 5 in Figure 11, which covers more than three consecutive 


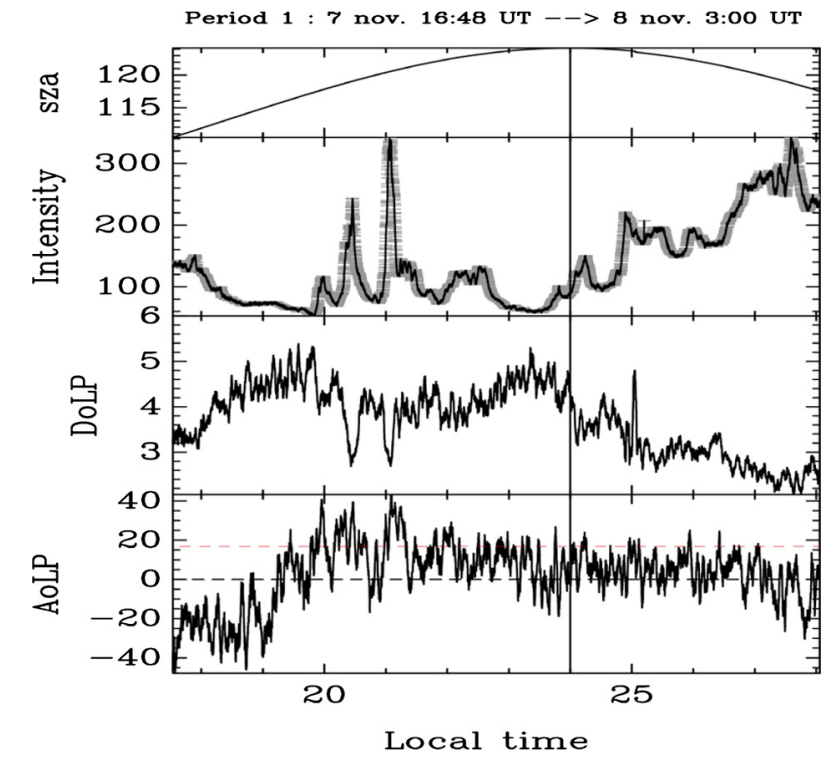

Fig. 7. Upper panel: solar zenith angle in degrees. Lower panel: total intensity of the red line in count rates (see text). The error bars are given in gray. The next two panels below show the DoLP in \% and the AoLP in degrees. $0^{\circ}$ is vertical and $90^{\circ}$ is horizontal. The dashed red line represents the measured instrumental AoLP. The vertical full line indicates midnight.

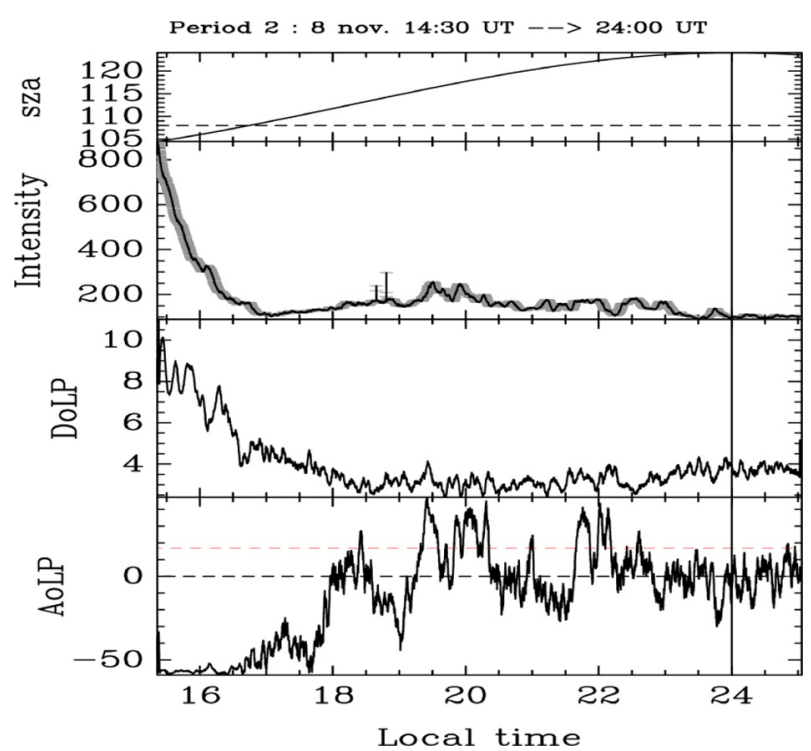

Fig. 8. Same as in Figure 7, for period 2. Upper panel: the dashed horizontal line shows the $108^{\circ}$ limit.

days. The DoLP exhibits a rather large scatter when the intensity of the red line is faint (less than 100 counts). For example, during period 4, between 20:00 LT and 1:00 LT the next day, it ranges from $\sim 4 \%$ to $6 \%$. A maximum of around $7 \%$ is observed during the night between December 6 and 7 (period 5). This maximum corresponds also to a period with an intensity smaller than 100 counts but the variations of the AoLP ensure that this polarization is not due to noise. When there is an aurora, which strongly increases the intensity of the red line (see e.g. period 4 between 2:00 LT and 5:00 LT), the DoLP strongly decreases to values of around $2 \%$. This was already observed during previous campaigns and is confirmed here.

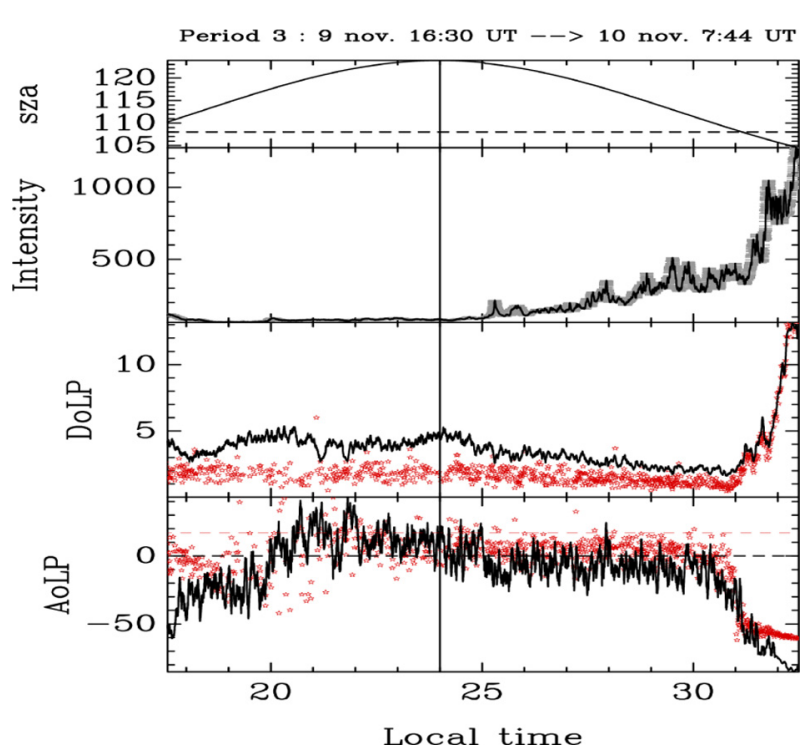

Fig. 9. Same as in Figure 7, for period 3. Notice that for November 9 , the statistics shows that the polarization should be regarded with more care. The red crosses show the result of the averaging data process.

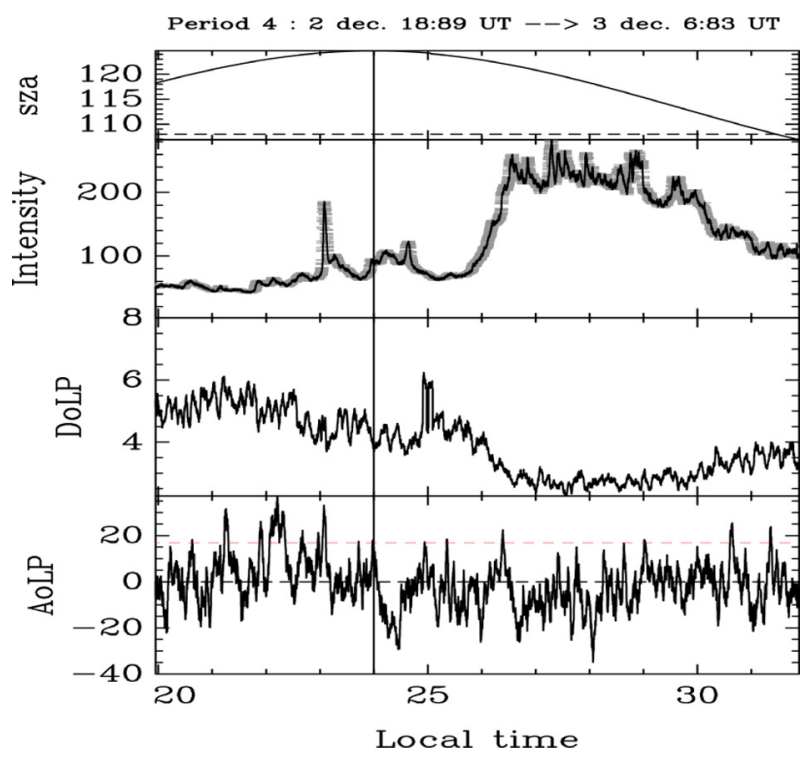

Fig. 10. Same as in Figure 7, for period 4.

To better quantitatively estimate these variations, the values of the DoLP are plotted versus the intensity of the red line in Figure 13 . Only data obtained at night $\left(\mathrm{SZA}>108^{\circ}\right)$ during all periods are considered, thus preventing any contribution from Rayleigh polarization of sunlight. The DoLP ranges from $\sim 2 \%$ to $\sim 7 \%$. These values are much larger than the instrumental polarization and ensure that most of the polarization observed is intrinsic.

The range of the DoLP decreases when the intensity increases. This result is encouraging to try to use the DoLP as a proxy for geomagnetic conditions. However, before drawing any conclusion, additional observations especially during more active geomagnetically conditions are necessary. Indeed during this campaign, the geomagnetic conditions were too 


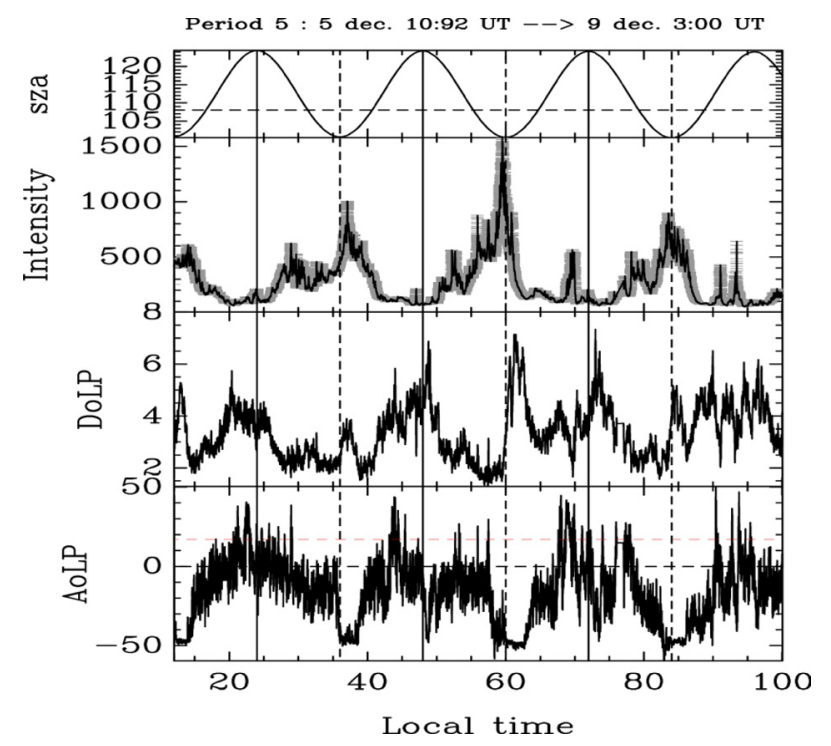

Fig. 11. Same as in Figure 7, for period 5. The vertical dashed line shows 12:00 LT. Notice that for December 9, the statistics shows that the polarization should be regarded with more care.

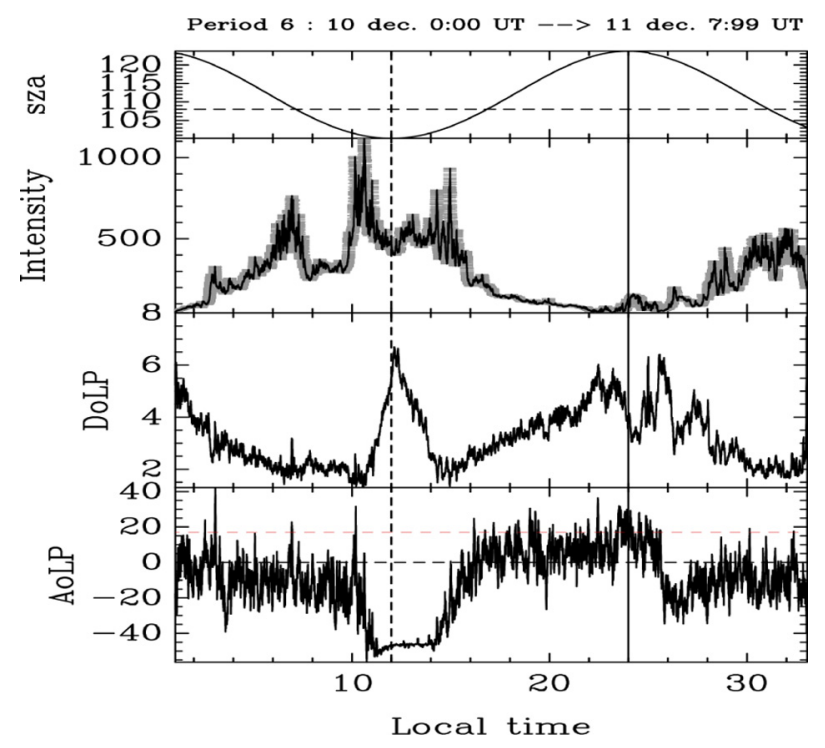

Fig. 12. Same as in Figure 7, for period 6.

homogeneous. However, it is worth noticing an interesting feature. The $A p$ index reaches a maximum on December 7. In Figure 11 , the DoLP maximizes at a value of $\sim 7 \%$ on the same day at 1:30 LT. This is the maximum value obtained at night over the whole dataset. It occurred while the red line intensity remained small and almost constant for a few hours. This may be a clue that the DoLP could be sensitive to the geomagnetic activity.

\subsection{Angle of linear polarization (AoLP)}

In order to calibrate the AoLP, we measured the polarization of a computer screen (Mac Book Pro). From a calibrated analyzer, we verified that this direction is $-45^{\circ} \pm 1^{\circ}$ (the sign being as in the trigonometric circle). The two processing methods retrieve this polarization angle.

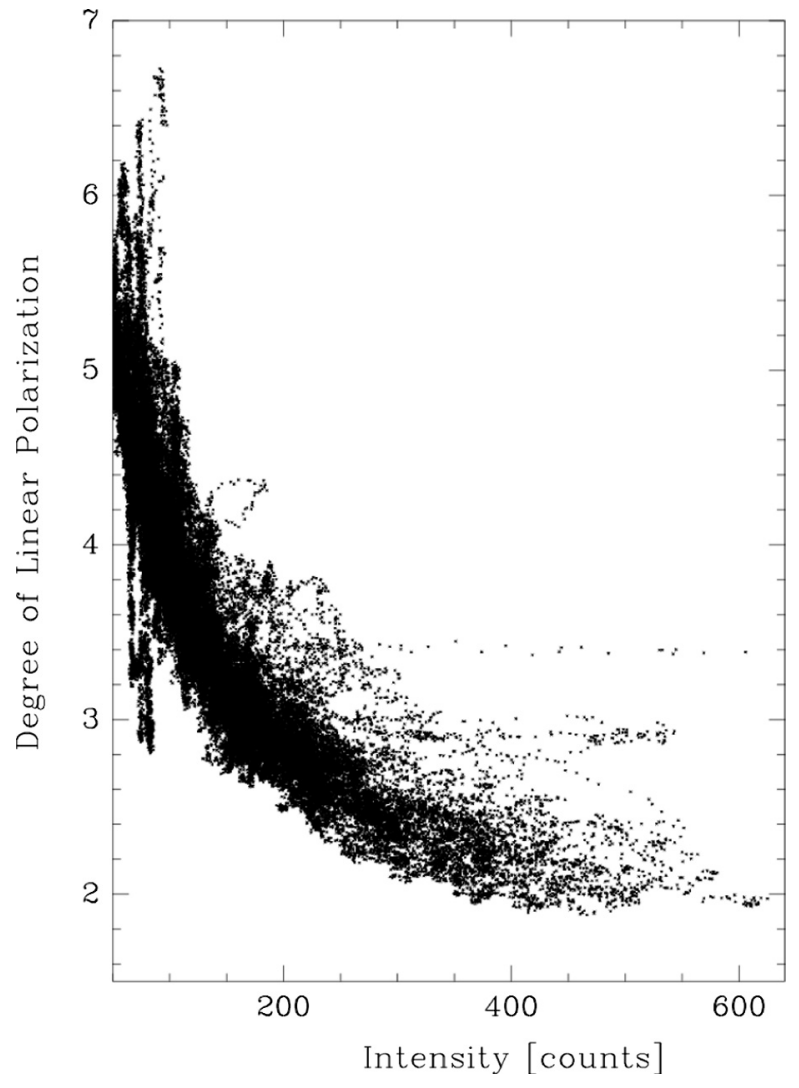

Fig. 13. Night DoLP (\%) versus the intensity. We checked that this global behavior does not depend on the time span around midnight as soon as the solar zenith angle is larger than $108^{\circ}$.

Because we point perpendicular to the magnetic field at low elevation in one direction only, we see the AoLP projected on the sky as on a screen, i.e. in two dimensions. An AoLP of zero degree corresponds to the vertical.

In Figure 9, we also present a comparison between the two methods for the AoLP. The behavior is the same of course, although there is more dispersion with the averaging method. Interestingly, the values are the same, and no trend is seen in any processing. Therefore, we only provide the AoLP with the filtering method in the other periods. During the day (i.e. when the solar zenith angle is smaller than $108^{\circ}$ ), the dominant polarizing mechanism is the multiple Rayleigh scattering. The AoLP becomes systematically negative (in the actual convention). The maximum amplitude observed during the 4 days when observations are available at noon is $-45^{\circ}$. The variation from one day to the other seems to indicate that with more data, it may be possible to infer the influence of the geomagnetic activity on the thermosphere from the polarization but again, since the major contributor is probably the aerosols, this will necessitate a large modeling effort.

The AoLP at night is close to the vertical during several hours in periods 2 and 4 and also during several night spans in period 5. During period 6, the AoLP is vertical the first night. During the second night, it remains slightly positive until about midnight. Shortly after local midnight, it turns almost exactly vertical. The same pattern occurs during period 3. Again, the sparsity of the data prevents us from drawing general conclusions. The fact that the AoLP at night is close to zero, corresponding to a full alignment with the magnetic field line, is 


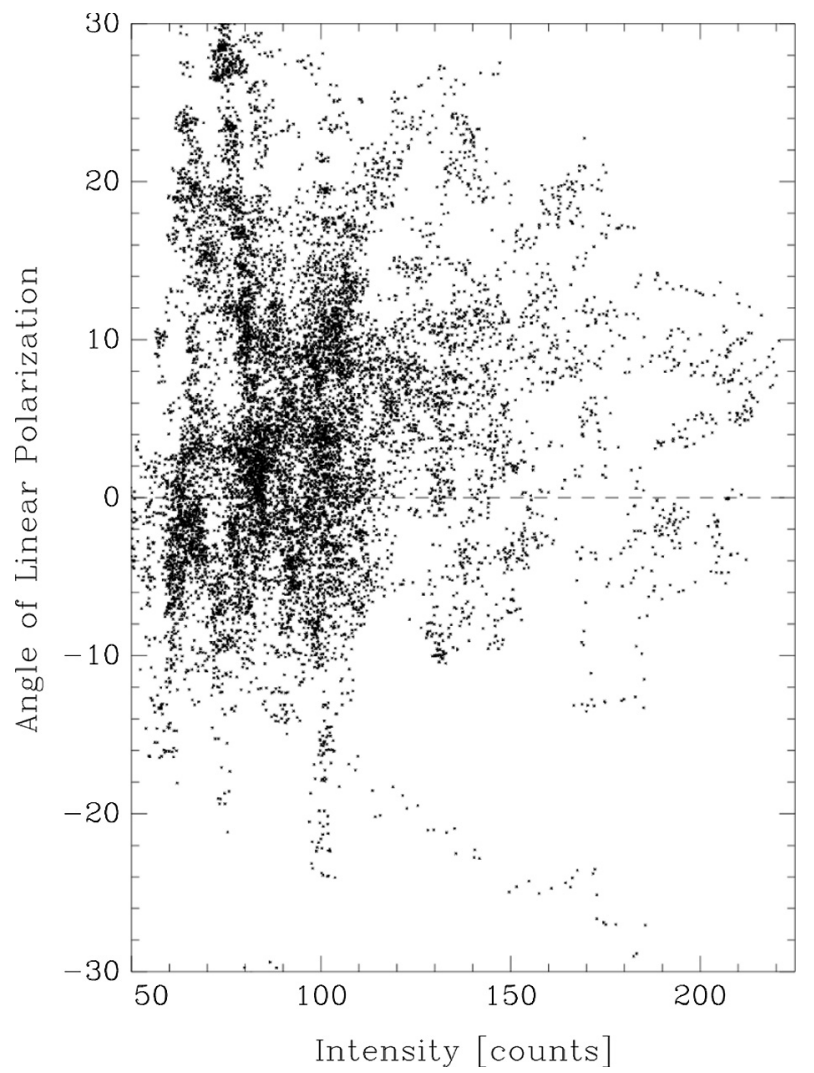

Fig. 14. AoLP (in degrees) versus the intensity, $\pm 1 \mathrm{~h}$ around local midnight.

in agreement with the theoretical prediction of Bommier et al. (2010). However, the average value over all the nights is not exactly zero but about $2.2^{\circ}$. This may indicate that we are not pointing absolutely perpendicular to the magnetic field, or that the actual field lines in the pointing direction are slightly tilted. This is however quite different from the previous experiments at Longyearbyen where a $20^{\circ}$ (i.e. Eastward-directed) AoLP was found. A possible explanation of this shift of the AoLP is a contamination by a light pollution component.

The AoLP is also affected by the intensity of the red line during the night. In several cases, it turns positive when the intensity increases and comes back to zero when the aurora is faint. A good example may be seen during the period 2 at 19:30 and 20:00 LT: two small increases in the intensity result in the variation of the AoLP (while the DoLP decrease in coordination). However, this is not a systematic trend: for low intensities, the directions range from -20 to $+20^{\circ}$. The geomagnetic activity seems to influence the AoLP as well. Coming back to the December 7 (period 5) with an $A p$ of 7, we see that when the DoLP maximizes at $1 \mathrm{LT}$, the angle turns negative.

In Figure 14, we plot the AoLP versus the intensity around local midnight $\left( \pm 1 \mathrm{~h}\right.$ ). It is distributed around $0^{\circ}$ (i.e. parallel to the magnetic field line) for low intensity. For high intensities, the AoLP seems to narrow around zero but here again, it is dangerous to draw further conclusions because of the small number of hours in the database $(<200)$. The variation of the AoLP clearly indicates that the measured signal is not pure noise. Would it be so, the measured AoLP would be of any value between $-90^{\circ}$ and $+90^{\circ}$.

\section{Discussion and conclusion}

The polarization has been measured in a polar station, without any artificial light. The main results are:

- We confirmed that the atomic oxygen red line is partially polarized and thus the light pollution is not the only polarization component in Lilensten et al. (2008) and in Barthélemy et al. (2011).

- IThe DoLP ranges between $2 \%$ and $7 \%$. This value is much smaller than the $17 \%$ theoretically predicted by Bommier et al. (2010). This might indicate a depolarization effect due to atmospheric collisions, as already pointed out by Chamberlain (1959). Since these collisions depend on the density, it raises some hope to use the polarization as a neutral atmospheric density proxy. The red line DoLP is larger when the intensity is fainter, confirming the first observations. This anti-correlation can be interpreted as follows: electrons from the polar rain, with a mean energy of several hundreds of electronvolts, constantly precipitate in the atmosphere, resulting in a permanent polarization of the red line emission. These electrons are at the origin of the background polarization that is continuously observed with the instrument. During an auroral event, the electron fluxes around $500 \mathrm{eV}$ increase, resulting in an enhancement of the red line intensity and of the difference $Q=I_{\max }-I_{\min }$. However, the sum $I_{\max }+I_{\min }$ increases more than $Q$. The DoLP $P$ is the ratio of these two quantities and is therefore decreasing. Moreover, during an auroral event, there is also additional high-energy electron precipitation. If the latter creates less polarization, the ratio $P$ will decrease while $Q$ increases. In Bommier et al. (2010), the DoLP is independent from the electron energy. This seems to contradict our interpretation. However, highenergy electrons do not only excite, but mainly ionize the ambient gas with more efficiency.

- The nighttime AoLP is close to the direction of the magnetic field line but it fluctuates during periods of low intensity. This is in good agreement with Bommier et al. (2010). In their conclusion, they suggest that without auroral events, the observed polarization is either of instrumental origin or due to scattered parasitic light. There is no parasitic light in Hornsund and the fact that the AoLP does not remain constant does not support an instrumental origin. The actual data processing using the Mueller matrix clears the data from this artifact. Moreover, the mere fact that the AoLP is always $0 \pm 20^{\circ}$ when the sun is low enough is direct evidence that the DoLP measurement is real. Therefore, we believe that the observations are not due to instrumental polarization. When averaged at night, the AoLP is not exactly vertical but close to $2.2^{\circ}$. We have no clear explanation to this feature, which can be simply caused by the fact that the angle between the instrument and the magnetic field is not exactly perpendicular.

- One goal of this experimental campaign was to link the polarization to the geomagnetic activity. Unfortunately, severe polar conditions caused the instrument to fail after a few weeks of operation and it has not been possible to recover it. Since the geomagnetic conditions were very stable during the operating periods (with less than 200 useable hours), it is not possible to correlate the polarization parameters to the geomagnetic activity. However, the 


\section{J. Lilensten et al.: Polarization of the auroral red line}

AoLP is close to the vertical and from these first statistics, the DoLP decreases in a coherent manner when the intensity of the red line increases.

A new spectropolarimeter is currently being built, which will allow to measure the polarization across the auroral spectrum from $\sim 400 \mathrm{~nm}$ to $\sim 700 \mathrm{~nm}$. This instrument will run in parallel to the present SPP so as to have two independent measurements. Observations are foreseen in 2012/2013. In parallel, two complementary theoretical studies will be undertaken: first, the theoretical polarization will be computed taking into account some depolarizing mechanisms such as the collisions or the chemical reactions at the origin of the $\mathrm{O}^{1} \mathrm{D}$ excited state. The second study will consist in evaluating the dayside theoretical Rayleigh scattering in order to deduce the component due to the auroral activity.

Acknowledgements. We are very thankful to the whole Hornsund staff for helping with so much enthusiasm to install SPP in their marvelous base for the next winter. We thank the Hornsund Polish Polar Station for using GLACIO-TOPOCLIM service. The present research project has been supported by the French Polar Institute (IPEV No. 1026) and by the European project COST ES0803 "Developing space weather products and services in Europe". It is also part of the European project COST MP1104 "Polarization as a tool to study the Solar System and beyond" and the European FP7 Project ESPAS, Grant Agreement No. 283676. We thank S. Maret and S. Bardeau for helping us in computer fixing. This project has been funded by the European FP7 ESPAS action (Near-Earth space data infrastructure for e-Science).

\section{References}

Barthélemy, M., J. Lilensten, F. Pitout, C. Simon-Wedlund, R. Thissen, et al. Polarisation in the auroral red line during coordinated EISCAT Svalbard Radar/optical experiments, Ann. Geophys., 29, 1101-1112, DOI: 10.5194/angeo-29-1101-2011, 2011.

Bommier, V., S. Sahal-Brechot, J. Dubau, and M. Cornille, The theoretical impact polarization of the O I 6300 A red line of Earth aurorae, Ann. Geophys., 29, 71-79, DOI: 10.5194/angeo-29-71-2011, 2010.

Chamberlain, J., On the polarization of the oxygen red line in aurorae, Planet. Space Sci., 2, 73-75, 1959.

Clarke, D., Stellar Polarimetry, Wiley. ISBN: 978-3-527-40895-5, 2010.

Cronin, T.W., E.J. Warrant, and B. Greiner, Celestial polarization patterns during twilight, Appl. Opt., 45, 5582-5589, 2006.

Fairfield, D.H., and J.D. Scudder, : Polar rain - Solar coronal electrons in the earth's magnetosphere, J. Geophys. Res., 90, 4055-4068, DOI: 10.1029/JA090iA05p04055, 1985.
Foord, R., C. Jones, J. Oliver, and E.R. Pike, The use of photomultiplier tubes for photon counting, Appl. Opt., 8, 19751989, DOI: 10.1029/JA090iA05p04055, 1969.

Goldstein, D., Polarized light, 2nd edn, Marcel Dekker, New York, NY, 2003.

Hamamatsu Technical Information, Photon counting using photomultiplier tubes, Hamamatsu Photonics K.K., Japan, 1998.

Johnsen, M.G., D.A. Lorentzen, J.M. Holmes, and U.P. Løvhaug, A model based method for obtaining the open/closed field line boundary from the cusp auroral $6300 \AA[\mathrm{OI}]$ red line, J. Geophys. Res. (Space Phys.), 117, A03319, DOI: 10.1029/2011JA016980, 2012.

Lagarias, J.C., J.A. Reeds, M.H. Wright, and P.E. Wright, Convergence properties of the Nelder-Mead simplex method in low dimensions, SIAM J. Optim., 9, 112-147, 1998.

Lilensten, J., C. Simon, M. Barthélemy, J. Moen, R. Thissen, and D.A. Lorentzen, Measurement of the polarization of the oxygen thermospheric red line: a review and a prospective in the frame of Space Weather studies, Space Weather, 4 , DOI: 10.1029/2006SW000228, 2006.

Lilensten, J., J. Moen, M. Barthélemy, R. Thissen, C. Simon, D.A. Lorentzen, O. Dutuit, P.O. Amblard, and F. Sigernes, Polarization in aurorae: A new dimension for space environments studies, Geophys. Res. Lett., 35, 8804, DOI: 10.1029/2007GL033006, 2008.

Lorentzen, D.A., N. Shumilov, and J. Moen, Drifting airglow patches in relation to tail reconnection, Geophys. Res. Lett., 31, L02 806, DOI: 10.1029/2003GL017785, 2004.

Martinez Herrero, R., P. Mejias, and G. Piquero, Characterization of partially polarized light field, Springer Publishing, Berlin. ISBN: 978-3-642-01326-3, 2009.

Moen, J., N. Gulbrandsen, D.A. Lorentzen, and H. Carlson, On the MLT distribution of F-region polar cap patches at night, Geophys. Res. Lett., 34, L14113, DOI: 10.1029/2007GL029632, 2007.

Moen, J., X. Qiu, H. Carlson, R. Fujii, and I. McCrea, On the diurnal variability in F2-region plasma density above the EISCAT Svalbard radar, Ann. Geophys., 26, 2427-2433,

DOI: 10.5194/angeo-26-2427-2008, 2008.

Newell, P.T., and Meng, Intense keV energy polar rain, J. Geophys. Res., 95, 7869-7879, 1990.

Press, W.H., S.A. Teukolsky, W.T. Vetterling, and B.P. Flannery, Numerical recipes in Fortran. The art of scientific computing, 2nd ed., Cambridge University Press, New York, 1992.

Stenflo, J., Solar Magnetic Fields, vol. 189, Kluwer Academic Pub., Astrophysics and Space Science Library (ASSL), 1994.

Ugolnikova, O.S., O.V. Postylyakovb, and I.A. Maslova, Effects of multiple scattering and atmospheric aerosol on the polarization of the twilight sky, J. Quant. Spectrosc. Radiat. Transf., 88, 233241, 2004.

Witasse, O., J. Lilensten, C. Lathuillere, and B. Pibaret, Meridional thermospheric neutral wind at high latitude over a full solar cycle, Ann. Geophys., 16, 1400-1409, DOI: 10.1007/s005850050706, 1998. 


\section{Appendix A: Environmental conditions}

\section{A.1. Meteorology}

Table 3 is a summary of the weather conditions above Hornsund. During the first time span (November 7 to November 9 ), the mean temperature decreases from $-12.3{ }^{\circ} \mathrm{C}$ to $-12.9^{\circ} \mathrm{C}$. The 10 was slightly less cold with maximum temperatures of $-9.5^{\circ} \mathrm{C}$. The sky was cloudy on November 6 but during the next 2 days, clouds were observed only around 15 UT. From the 7 to the 9 there was no precipitation but on November 10, $4 \mathrm{~mm}$ of fall was registered. On December the 2nd and 3rd between 00:00 and 06:00 and on December the 4 th, the visibility decreased drastically, while clouds covered much of the sky on December the 2nd and 3rd. Some precipitation was detected during December 6th, and between December 5 and 11 the sky was almost cloudless except a short time of scale (the order of hour) occasional overcast. Between December the 2 nd and 6 th, the mean temperature was around $-12^{\circ} \mathrm{C}$. The temperature decreased from December 6th to 10th (minimum temperature of $-13.6{ }^{\circ} \mathrm{C}$ to $-19.6{ }^{\circ} \mathrm{C}$ ) and from there it increased drastically (mean temperature $-5.9^{\circ} \mathrm{C}$ the December 11). However from the December 8 th the pressure increased continuously up to $1014.5 \mathrm{mb}$ on the December 11, but during all this period the visibility was very high.

Because we point toward the north, we also analyzed the Longyearbyen weather conditions as recorded at the local meteorological station. Longyearbyen is located about $150 \mathrm{~km}$ North of Hornsund. The Longyearbyen weather conditions are very similar to the one recorded at Hornsund.

\section{A.2. Geophysics}

Table 4 is a summary of the solar and geomagnetic activity during the clear days for which we have good polarization measurements. We also show the solar conditions for the 3 days before these periods in order to account for the time necessary for the solar wind to reach the Earth. The following observations are based on the NOAA and STCE bulletins. We also make use of the KHO/Unis (University Centre in Svalbard) local observations.

\section{A.2.1. Solar activity}

In November, the solar activity changed from moderate to high level and then decreased to very low levels. A C-class X-ray flare, a M-class X-ray flare, and an optical sub-flare were observed on November 4. A C-class X-ray flare, a M-class $\mathrm{X}$-ray flare, and two optical sub-flares were observed on November 5. Four C-class X-ray flares, a M-class X-ray flare, two optical sub-flares, and one optical flare occurred on November 6. Only a C-class X-ray flare and one optical flare happened on November 7. There were five optical sub-flares on November 10 . No proton events were observed at geosynchronous orbit. The greater than $2 \mathrm{MeV}$ electron flux at geosynchronous orbit was at normal to moderate levels.

The solar activity was at very low levels at the end of November and beginning of December. Only one C-class Xray flare was observed on December 1 . No proton event was observed at geosynchronous orbit. The greater than $2 \mathrm{MeV}$ electron flux at geosynchronous orbit was at low levels.

From December 5 to 11, the solar activity was at very low levels. There was a unipolar spot group on December 2 and 4.
Table 3. Weather conditions over Hornsund. The first three columns give the temperatures in ${ }^{\circ} \mathrm{C}$. The pressure $P$ is in mb. Precipitations are in $\mathrm{mm}$.

\begin{tabular}{lrrrrl}
\hline \multicolumn{1}{c}{ Date } & Mean & \multicolumn{1}{c}{ Max } & Min & \multicolumn{1}{c}{$P$} & Prec \\
\hline Nov. 7 & -12.3 & -9.5 & -14.7 & 997.4 & 0 \\
Nov. 8 & -12.2 & -11.0 & -14.3 & 1000.7 & 0 \\
Nov. 9 & -12.9 & -11.5 & -15.4 & 1004.8 & 0 \\
Nov. 10 & -11.6 & -9.5 & -14.3 & 1009.4 & 0.4 \\
Dec. 2 & -11.8 & -6.3 & -13.7 & $989 / 998.9$ & 0 \\
Dec. 3 & -11.9 & -10.1 & -14.9 & 1006.3 & 0.6 \\
Dec. 5 & -11.1 & -10.1 & -13.6 & 1006.6 & 0 \\
Dec. 6 & -12.5 & -11.2 & -13.6 & 999.6 & 0 \\
Dec. 7 & -15.0 & -12.2 & -17.2 & 998.8 & 0 \\
Dec. 8 & -16.2 & -13.9 & -19.0 & 1002.0 & 0 \\
Dec. 9 & -16.4 & -14.5 & -18.4 & 1008.7 & 0 \\
Dec. 10 & -16.1 & -12.4 & -19.6 & 1012.3 & 0 \\
Dec. 11 & -5.6 & -3.9 & -13.9 & 1014.4 & 0 \\
\hline
\end{tabular}

Table 4. Solar and geomagnetic conditions. The Lyman $\alpha$ flux is in $10^{11}$ photons $\mathrm{cm}^{-2} \mathrm{~s}^{-1}$.

\begin{tabular}{lcccc}
\hline \hline \multicolumn{1}{c}{ Date } & F10.7 & SSN & Ly $\alpha$ & $A p$ \\
\hline Nov. 4 & 79 & 34 & 3.53 & \\
Nov. 5 & 83 & 29 & 3.67 & \\
Nov. 6 & 89 & 43 & 3.82 & \\
Nov. 7 & 85 & 34 & 3.87 & 1 \\
Nov. 8 & 84 & 36 & 3.83 & 3 \\
Nov. 9 & 84 & 35 & 3.88 & 3 \\
Nov. 10 & 86 & 55 & 3.93 & 4 \\
Nov. 29 & 83 & 31 & 3.75 & \\
Nov. 30 & 86 & 24 & 3.74 & \\
Dec. 1 & 87 & 25 & 3.76 & \\
Dec. 2 & 87 & 32 & 3.79 & 3 \\
Dec. 3 & 87 & 27 & 3.83 & 1 \\
Dec. 4 & 87 & 48 & 3.85 & \\
Dec. 5 & 88 & 47 & 3.90 & 1 \\
Dec. 6 & 89 & 35 & 3.93 & 2 \\
Dec. 7 & 87 & 34 & 3.92 & 4 \\
Dec. 8 & 87 & 22 & 3.93 & 3 \\
Dec. 9 & 87 & 22 & 3.93 & 2 \\
Dec. 10 & 88 & 33 & 3.91 & 0 \\
Dec. 11 & 87 & 25 & 3.93 & 1 \\
\hline
\end{tabular}

A large filament eruption was observed on December 6 and there was also an associated CME, which was not forecast to become geoeffective. No proton events were observed at geosynchronous orbit. The greater than $2 \mathrm{MeV}$ electron flux at geosynchronous orbit was at low levels and moved to normal levels from December 6.

\section{A.2.2. Geomagnetic activity}

Comparison with the local geomagnetic activity in Hornsund is not relevant. Indeed, with the pointing angle and elevation of the SPP, the observed red emission occurs more than $400 \mathrm{~km}$ a way to the north, in the Arctic ocean, where no measurement of the local geomagnetic activity is available. Therefore, we can only compare with planetary observations.

Geomagnetic field activity was mostly quiet between November 7 and 10. Activity increased to moderate levels late on November 10 as a CME passage began to disturb the geomagnetic field. A variation of the geomagnetic activity in 
Longyearbyen occurred on November 7 between 19:00 UT and 22:00 UT with $D$-component and $Z$-component variations (with a peak of $110 \mathrm{nT}$ for the $Z$-component and $90 \mathrm{nT}$ for the $D$-component). Another variation occurred on November 8 and 9 between 12:00 UT and 02:00 UT (a peak larger than $110 \mathrm{nT}$ for the $H$-component and one around $95 \mathrm{nT}$ for the $Z$-component). On November 10 , there was a $Z$-component variation between 18:00 UT and 24:00 UT with a peak intensity of more than $110 \mathrm{nT}$.

Geomagnetic activity was predominantly quiet for the entire period ranging from November 29 to December 3. Highest level for $K p$ index was observed on December 2 and was only equal to 2 . The greatest variation of the geomagnetic activity in Longyearbyen took place on December 2 between 1:00 UT and 8:00 UT (a peak of $110 \mathrm{nT}$ for the $Z$-component variation). A lower variation of the $Z$-component (around $60 \mathrm{nT}$ ) has been noticed between 21:00 and 23:30.

Geomagnetic field activity was at predominantly quiet levels from December 5 to 11. A variation of the geomagnetic activity in Longyearbyen happened on December 5 and 6 between 22:00 UT and 2:00 UT (a peak of more than $110 \mathrm{nT}$ for the $Z$-component variation). Another one with the same magnitude took place on December 7 and 8 from 19:00 UT to 3:00 UT. There was also a replica on December 8 and December 9 between 19:20 UT and 4:00 UT, which had a peak intensity similar to the previous ones. A lower variation of the Z-component (around $80 \mathrm{nT}$ ) was noticed between 21:00 UT and 23:00 UT on December 11.

\section{A.2.3. Auroral activity}

In order to detect auroral periods, we used the all sky cameras from the KHO observatory located $15 \mathrm{~km}$ south of Longyearbyen. These cameras cover all the sky above the Svalbard archipelago in the red and green lines. On November 7, there were auroras from 18:50 UT to 24:00 UT. On November 8, auroras were observed from 17:00 UT to 23:30 UT with the brightest ones at 18:00 UT. On November 10, there were bright auroras between 17:20 UT and 21:30 UT. On December 2, bright auroras were observed from 3:00 UT to 8:00 UT and fainter ones from 21:00 UT to 23:30 UT. There were a few auroras on December 3 between 00:00 UT and 08:00 UT. Finally, a faint aurora occurred on December 5 from 1:20 UT to 1:50 UT. Discrete auroras were then observed until daylight on December 6, as well as between December 6 and 7 from 19:00 UT to daylight. During the night of December 7, a huge auroral activity lasted from the sunset to the middle of the night. This auroral activity remained on December 8 although with a lesser extent, and lasted until the end of the period.

\section{Appendix B: Removal of the spikes in the data}

As winter went forward, the polarized channel SPP deteriorated. As a consequence, periodical bursts of spikes decorated the counting measure provided by the channel. In order to eliminate these spurious spikes in an automated way, we develop the following method. The data for one session are cut into temporal blocks of size 256 samples. This size is justified by the fact that it is the first power of two containing two periods, and that it was found adequate for the first task described below. For each block and each angle, the following steps are performed.
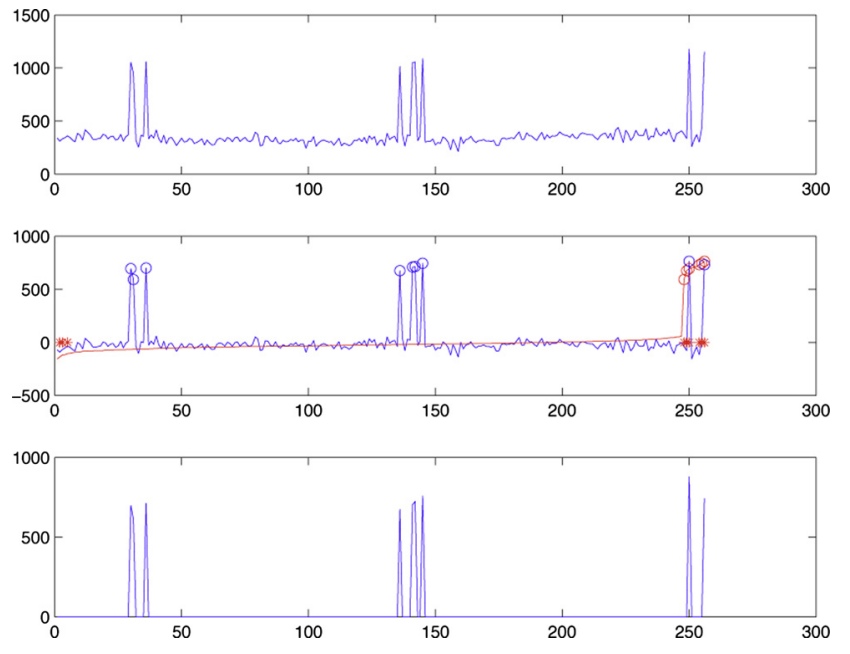

Fig. 15. The upper panel shows an example of a signal affected with spikes. The time here is not important. In the middle panel, the spikes have been automatically identified and are extracted in the bottom panel.

1. Detrend. We detrend the data using an orthonormal wavelet decomposition, setting the approximation to zero in the wavelet decomposition. We used six analyzed bands for this wavelet decomposition since it provides the best result. We verified that the choice of the wavelet had no influence on the result by checking the polarization between the periods with spike and within the corrected periods: they show no irregularities statistically larger than in the clean periods. Thus a Daubechies 2 wavelet have been used.

2. Sorting. Each block may contain up to three bursts of one or some spikes. These spikes are in general from some tens up to several hundreds of units. Since they are very well localized and that there are from some spikes up to a dozen per block, a simple idea is to sort the data in the block: the sorted data will show an increase at low mean rate followed by a dramatic change in the increase rate once the first spike is considered in the sorting. We then estimate the position of the sudden first big increase in the sorted data. All the following sorted data are thus belonging to spikes, and we have located their indices in the block. To decide for the first spike, we set up a threshold of 10 units. Furthermore, since there are less than 20 spikes per block, we search for increase of more than 10 units only in the second half of the sorted data. This eliminates possible false detections.

3. Interpolation. Once we have located the indices of the spikes, we suppress them by a simple linear interpolation.

Figure 15 shows the result of this denoising on a example.

\section{Appendix C: Correction of the instrumental polarization}

The SPP (photo-multiplier + polarizing filter) introduces instrumental polarization and modifies the state of the polarization of the incident light. To remove the effect of the instrumental polarization, the Mueller formalism (see e.g. Stenflo 1994; Goldstein 2003; Martinez Herrero et al. 2009) is used. Mueller matrix can handle propagation of partially polarized light 
through optical systems. It transforms the Stokes vector by successive multiplications with $4 \times 4$ matrices belonging to individual optical elements. In our case, the SPP is considered as a partial polarizer whose Mueller matrix $\mathbf{M}_{\mathbf{4}}$ is (e.g. Clarke 2010)

$$
\mathbf{M}_{4}=\frac{1}{2}\left(\begin{array}{cccc}
K_{x}+K_{y} & K_{x}-K_{y} & 0 & 0 \\
K_{x}-K_{y} & K_{x}+K_{y} & 0 & 0 \\
0 & 0 & 2 \sqrt{K_{x} K_{y}} & 0 \\
0 & 0 & 0 & 2 \sqrt{K_{x} K_{y}}
\end{array}\right),
$$

where, $K_{x}$ and $K_{y}$ are the intensity transmission coefficients of SPP in two orthogonal directions $x$ and $y$. The $x$-axis is chosen along the direction of the instrumental polarization such that $\theta_{\mathrm{i}}=0$. The $z$-axis is along the optical axis of the instrument, and the $y$-axis completes the right-handed orthogonal frame. If $P_{\mathrm{i}}$ is defined as $\left(K_{x}-K_{y}\right) /\left(K_{x}+K_{y}\right), \mathbf{M}_{\mathbf{4}}$ becomes

$$
\mathbf{M}_{4}=\frac{K_{x}+K_{y}}{2}\left(\begin{array}{cccc}
1 & P_{\mathrm{i}} & 0 & 0 \\
P_{\mathrm{i}} & 1 & 0 & 0 \\
0 & 0 & \sqrt{1-P_{\mathrm{i}}^{2}} & 0 \\
0 & 0 & 0 & \sqrt{1-P_{\mathrm{i}}^{2}}
\end{array}\right) .
$$

In the following, the index o stands for "observed", $\mathrm{r}$ for "real", and i for "instrumental". $P$ is the DoLP, $\theta$ is the AoLP. The initial state of polarization, hereafter referred to as "real", is described by the Stokes vector $\mathbf{S}_{\mathbf{r}}$

$$
\mathbf{S}_{\mathbf{r}}=\left(\begin{array}{c}
I_{\mathrm{r}} \\
Q_{\mathrm{r}} \\
U_{\mathrm{r}} \\
V_{\mathrm{r}}
\end{array}\right)
$$

while the observed polarization is described by the Stokes vector $\mathbf{S}_{\mathbf{o}}$

$$
\mathbf{S}_{\mathbf{0}}=\left(\begin{array}{c}
1 \\
P_{\mathrm{o}} \\
0 \\
0
\end{array}\right)
$$

Circular polarization is null because a linear polarizer is used $\left(V_{\mathrm{o}}=0\right)$. Along the observed direction of polarization $\theta_{\mathrm{o}}$ chosen as reference axis for the linear polarization, $P$ is entirely contained in the $Q$ Stokes parameter, hence $Q_{\mathrm{o}}=P_{\mathrm{o}}$. The relation between $\mathbf{S}_{\mathbf{o}}$ and $\mathbf{S}_{\mathbf{r}}$ is given by

$$
\mathbf{S}_{\mathrm{o}}=\mathbf{R}_{\mathbf{4}}^{-1} \mathbf{M}_{4} \mathbf{R}_{4} \mathbf{S}_{\mathrm{r}}
$$

where $\mathbf{R}_{\mathbf{4}}$ is the rotation matrix

$$
\mathbf{R}_{4}=\left(\begin{array}{cccc}
1 & 0 & 0 & 0 \\
0 & \cos 2 \alpha & \sin 2 \alpha & 0 \\
0 & -\sin 2 \alpha & \cos 2 \alpha & 0 \\
0 & 0 & 0 & 1
\end{array}\right)
$$

which performs a rotation from the initial reference frame (where $\mathbf{S}_{\mathbf{r}}$ is expressed) into the frame corresponding to the partial polarizer where the $\mathbf{M}_{\mathbf{4}}$ Mueller matrix can be applied. The application of $\mathbf{R}_{\mathbf{4}}{ }^{-1}$ rotates the resulting Stokes vector back to the original reference frame and provides $\mathbf{S}_{\mathbf{0}} . \alpha$ is the angle between $\theta_{\mathrm{o}}$ and $\theta_{\mathrm{i}}$. Positive $\alpha$ is measured counterclockwise from the $x$-axis toward the $y$-axis as seen looking against the $z$ direction. In writing Eq. (A.1), we consider that the polarimeter as a whole adds a linear polarization of rate $P_{\mathrm{i}}$ and direction $\theta_{\mathrm{i}}$, which is described by the Mueller matrix $\mathbf{M}_{\mathbf{4}}$, to the incoming linear polarization of rate $P_{\mathrm{r}}$ and direction $\theta_{\mathrm{r}}$. The real Stokes vector $\mathbf{S}_{\mathbf{r}}$ can be obtained by

$$
S_{r}=R_{4}^{-1} M_{4}^{-1} R_{4} S_{0}=\Gamma_{4} S_{0}
$$

where the inverse of $\mathbf{M}_{\mathbf{4}}$ is

$$
\mathbf{M}_{4}^{-1}=\frac{K_{x}+K_{y}}{2 K_{x} K_{y}}\left(\begin{array}{cccc}
1 & -P_{\mathrm{i}} & 0 & 0 \\
-P_{\mathrm{i}} & 1 & 0 & 0 \\
0 & 0 & \sqrt{1-P_{\mathrm{i}}^{2}} & 0 \\
0 & 0 & 0 & \sqrt{1-P_{\mathrm{i}}^{2}}
\end{array}\right) .
$$

In order to simplify the expressions, we denote $S a=\sin 2 \alpha$, $C a=\cos 2 \alpha$, and $R=\sqrt{1-P_{\mathrm{i}}^{2}}$. The $T_{4}$ matrix becomes

$$
\Gamma_{4}=\frac{K_{x}+K_{y}}{2 K_{x} K_{y}}\left(\begin{array}{cccc}
1 & -C a P_{\mathrm{i}} & -S a P_{\mathrm{i}} & 0 \\
-C a P_{\mathrm{i}} & C a^{2}+S a^{2} R & C a S a(1-R) & 0 \\
0 & C a S a(1-R) & S a^{2}+C a^{2} R & 0 \\
0 & 0 & 0 & R
\end{array}\right) .
$$

The real DoLP is $P_{\mathrm{r}}=\frac{\sqrt{Q_{\mathrm{r}}^{2}+U_{\mathrm{r}}^{2}}}{I_{\mathrm{r}}}=\frac{N}{D}$ with

$$
N=\sqrt{P_{\mathrm{o}}^{2}+P_{\mathrm{i}}^{2}-2 P_{\mathrm{o}} P_{\mathrm{i}} C a-P_{\mathrm{o}}^{2} P_{\mathrm{i}}^{2} S a^{2}},
$$

and

$$
D=1-P_{\mathrm{o}} P_{\mathrm{i}} C a
$$

The real AoLP is deduced from the two following equations:

$$
\begin{aligned}
& \cos \left(2 \theta_{\mathrm{r}}\right)=\frac{\frac{O}{\mathrm{r}}_{\mathrm{r}}}{\frac{\mathrm{r}}{\mathrm{r}}_{\mathrm{r}}}=\frac{-\mathrm{Ca} P i+P_{\mathrm{o}} \mathrm{Ca}^{2}+P_{\mathrm{o}} S a^{2} \sqrt{1-P_{\mathrm{i}}^{2}}}{N}, \\
& \sin \left(2 \theta_{\mathrm{r}}\right)=\frac{U_{\mathrm{r}}}{\frac{T_{\mathrm{r}}}{P_{\mathrm{r}}}}=\frac{-S a P_{\mathrm{i}}+P_{\mathrm{o}} \mathrm{CaSa}\left(1-\sqrt{1-P_{\mathrm{i}}^{2}}\right)}{N},
\end{aligned}
$$

and applying the adequate quadrant correction according to the signs of $\cos \left(2 \theta_{\mathrm{r}}\right)$ and $\sin \left(2 \theta_{\mathrm{r}}\right)$. 